Enfoque epistemológico, teórico y metodológico de la categoría prácticas discursivas en la investigación en

Comunicación Social

Yamila Vazquez Bonne

https://perio.unlp.edu.ar/ojs/index.php/question/article/view/4850

Cita sugerida: Vazquez Bonne, Y. (2018). Enfoque epistemológico, teórico y metodológico de la categoría prácticas discursivas en la investigación en Comunicación Social. Question, 1(60), e107. doi:https://doi.org/10.24215/16696581e107

\title{
Enfoque epistemológico, teórico y metodológico de la categoría prácticas discursivas en la investigación en Comunicación Social
}

\author{
Epistemological, theoretical and methodological approach of the \\ discursive practices category in Social Communication research
}

Yamila Vazquez Bonne yvazquezbonne@gmail.com

http://orcid.org/0000-0002-4545-2866

Facultad de Comunicación; Universidad de La Habana (Cuba)

\section{Resumen}

En el estudio de la comunicación ha sido tradicional el interés por el análisis de las expresiones, cuyas denominaciones han sido diversas: mensaje, contenido, discurso, etcétera. Dentro de ellas, desde hace varios años ha ido cobrando auge la concepción de discurso como forma de organización de lo social o como práctica social, lo que determina la importancia que se le otorga en función de comprender la construcción de significados en las sociedades actuales. Teniendo como punto de partida un conjunto de problemáticas detectadas en una investigación realizada a la producción científica de la Facultad de Comunicación de la Universidad de La Habana, que emplea en análisis del discurso, el artículo realiza un 
acercamiento teórico a las categorías discurso y prácticas discursivas. Además propone implicaciones epistemológicas, teóricas y metodológicas de considerar la categoría prácticas discursivas para la investigación en el campo de la comunicación social. El abordaje del discurso desde la concepción de prácticas discursivas enriquece la perspectiva epistemológica, teórica y metodológica de la investigación en comunicación que debe repensarse a la luz de las demandas de la sociedad contemporánea, en la que las nuevas tecnologías asignan un papel activo a los sujetos y actores en los procesos discursivos y sociales.

Palabras clave: Discurso; prácticas discursivas; investigación; campo de comunicación social.

Abstract

In the study of communication has been traditional interest in the analysis of expressions, whose names have been diverse: message, content, discourse, etc. Within them, the conception of discourse as a form of organization of the social or as a social practice has been gaining momentum for several years, which determines the importance given to it in terms of understanding the construction of meanings in current societies. Taking as a starting point a set of problems detected in a research made to the scientific production of the School of Communication of the University of Havana, which uses in discourse analysis, the article makes a theoretical approach to the discourse and discursive practice categories. It also proposes epistemological, theoretical and methodological implications of considering the discursive practice category for research in the field of social communication. The approach to discourse from the conception of discursive practices enriches the epistemological, theoretical and methodological perspective of communication research that must be rethought in light of the demands of contemporary society, in which new technologies assign an active role to subjects and actors in the discursive and social processes.

Keywords: Discourse discursive practices; investigation; field of social communication.

El campo de la comunicación, cuyo espacio conceptual académico nace en el siglo XX (Galindo, 2008: XXII-XXV) se ha caracterizado por la convergencia de saberes provenientes de las nueve fuentes científicas de la comunicación: la Sociología Funcionalista, la Sociología Fenomenológica, la Psicología Social, la Semiótica, la Lingüística, la Sociología Critica, la 
Sociología cultural, la Economía Política y la Cibernética (Galindo, 2008: XXII-XXV). Ellas han constituido los cimientos teóricos y metodológicos que permiten explicar desde la investigación científica el funcionamiento de los componentes del proceso comunicativo: actores, expresiones, representaciones e instrumentos (Martín-Serrano, 1982: 100).

Destaca en este ámbito, el interés por el análisis de las expresiones cuya génesis data del período de surgimiento y auge de los medios de comunicación a partir de una mirada a la configuración de los mensajes producidos en estos espacios. Más allá de la pretensión por reconstruir una genealogía que explique cómo se ha conducido el estudio de las expresiones en el campo de la comunicación, lo que interesa en mayor medida es que, en los escenarios sociales de la contemporaneidad, se produce un cambio denotativo en referencia a la categoría en cuestión (mensaje, contenido, texto) que implica diferencias connotativas importantes. De ahí que adquiere relevancia no ya el mensaje, el código o el texto, sino el discurso, en tanto ente de organización de lo social.

Las concepciones investigativas acerca del funcionamiento de las sociedades contemporáneas se articulan con mayor impacto en la visión de que:

Las prácticas sociales se sustentan en el empleo de los discursos, pues poseen sentido en la comprensión de la imaginería que se produce en la sociedad y se convierten en un espacio de formación de imaginarios (...) a los que se recurre continuamente desde la visión de código compartido, sin el que no sería posible la comunicación" (Pérez, 2008: 227228).

El campo de la comunicación en sus prácticas profesionales, académicas e investigativas enfatiza en la producción y análisis de los discursos como resultado de su gestión en el espacio público y como fuente de información. Se prioriza este tipo de investigación en el ámbito especifico del periodismo, y mucho menos en el ejercicio del comunicador social.

Un estudio publicado en 2015 (Vázquez, 2015: 65) analiza el comportamiento de la producción científica (1) que declara emplear el análisis del discurso como técnica de investigación en la Facultad de Comunicación de la Universidad de La Habana (Cuba) durante el periodo comprendido entre 2005 y 2014. Como resultados de la indagación se pudo determinar la existencia de 82 investigaciones de pregrado y postgrado, devenidos en su mayoría de proceso de culminación de estudios de la Carrera de Periodismo (2). Se distribuyen en 17 los textos que responden a la culminación de estudios de la Carrera Comunicación Social y a la Maestría y Doctorado en Comunicación. 
Las temáticas más abordadas se engloban en los siguientes campos: estudios de género en la comunicación y el periodismo (literatura, medios impresos, audiovisual), estudios del discurso en relación con los procesos sociales en la comunicación y el periodismo (migración, política, etcétera). En este particular, se abordan fenómenos como los procesos migratorios, la construcción ideológica del discurso, la reproducción del poder, la hegemonía, entre otros. En todos los casos, el ámbito de la comunicación en que se sitúan es el masivo y las investigaciones tienen un corte fundamentado y comunicológico (Vázquez y Nápoles, 2015: 11).

Los objetos de estudios se centran en los siguientes soportes: la prensa escrita (editoriales, artículos periodísticos, etcétera); audiovisual: audiovisual informativo, audiovisual de ficción, documentales. En menor medida, se encontraron estudios acerca de revistas, cine, discursos políticos, radio, literatura (crónicas) y el discurso visual, específicamente el cartel (Vázquez y Nápoles, 2015: 11).

Desde el punto de vista teórico las definiciones empleadas en estas investigaciones sugieren que no existe consenso en la conceptualización de los términos discurso y análisis del discurso. El discurso y análisis del discurso son términos polisémicos; determinados por las perspectivas disciplinarias desde las cuales se han explicado estos conceptos. En la producción científica analizada, se asume una definición de discurso entendido como género, dígase: discurso periodístico, discurso audiovisual, discurso político, etcétera. Además, se conceptualiza como evento comunicativo y práctica social, compuesto por tres dimensiones: la cognitiva, la comunicativa y la sociocultural. Debido a que la perspectiva en comunicación social trasciende los análisis meramente lingüísticos, al considerar los discursos como procesos sociales, la manera de estudiarlos debe potenciar elementos de diversa naturaleza, como son el contexto de situación comunicativa, las condiciones de producción, circulación y recepción. Por otro lado, la conceptualización del análisis del discurso no logra establecer un consenso en torno a su estatuto científico, o sea si es técnica, método, metodología, teoría, etcétera (Vázquez, 2015: 104).

Dada la situación anterior, no hemos propuesto una investigación que contribuya a delinear los estudios en torno al discurso para el área de conocimientos de la Comunicación Social en Cuba. En aras de ello este artículo da cuenta de los primeros acercamientos al tema, desde lo que consideramos puede constituirse en desplazamiento analítico conceptual, que tiene implicaciones en el orden epistemológico, teórico y metodológico: extender la categoría discurso al de prácticas discursivas. Este artículo persigue dos propósitos: conceptualizar las nociones referidas a discurso y prácticas discursivas; y referir algunas de las implicaciones 
epistemológica, teórica y metodológica del desplazamiento del discurso al de prácticas discursivas en la investigación en el campo de la comunicación social.

La investigación tiene como antecedentes fundamentales las tesis de doctorado de las investigadoras cubanas Saladrigas (2005), Lugones (2016) y la tesis de Maestría de Vázquez (2015). En el ámbito internacional se fundamenta en los aportes de la investigadora Julieta Haidar (2000). Se adscribe al enfoque cualitativo, un tipo de investigación formativa que ofrece técnicas para obtener respuestas sobre las actitudes, creencias, motivos y comportamientos de los sujetos estudiados (Alonso y Saladrigas, 2002: 33). El análisis-síntesis y la revisión bibliográfica y documental constituyen los métodos y técnicas empleados.

\section{Del discurso y su relevancia social actual}

El término discurso presenta la peculiaridad de ser polisémico. Su conceptualización ha estado ligada a la disciplina académica que ha intentado su abordaje. En este sentido, ha sido definido con semejanza al texto, en tanto unidad superior de sentido mayor que la oración. Desde esta perspectiva (lingüística del texto) han sido asumidos los rasgos que caracterizan su construcción, especialmente la articulación de la coherencia y la cohesión desde el punto de vista formal; han sido descritas las maneras en que el significado se articula, por solo mencionar alguna de las aristas. El discurso ha sido visto como forma de uso real del lenguaje como forma de interacción social (van Dijk, 1992).

Entendido como evento comunicativo, el discurso parte de considerar no solo las condiciones que caracterizan al discurso a lo interno, o en otras palabras, los rasgos de su construcción lingüística, sino también comprende las afectaciones derivadas del contexto social en el que el discurso tiene lugar. De manera que son consideradas las dimensiones cognitivas, sociales y culturales en las que se emplea el lenguaje.

Desde la Pragmática ha sido visto equiparado con la situación de enunciación y se comprende a partir de la noción de actuación: ¿Qué hacemos cuando decimos algo? ¿Cuáles son las condiciones apropiadas, aceptables u oportunas en relación con las condiciones en que se produce la enunciación?

El investigador mexicano Tanius Karam (2005: 2) sistematiza las diversas definiciones de discurso a partir de 3 perspectivas: 
1. Formalista o intradiscursiva: discurso unidad lingüística superior a la oración con autores como Greimas y Harris;

2. Enunciativa: relación discurso (circunstancia de lugar y tiempo)-sujeto (encargado de la organización del lenguaje en función de)-destinatario;

3. Materialista: Discurso como práctica social, con autores como Pecheux y Robin.

En este último sentido se propone una definición de discurso que se ajusta de manera más coherente al área de la comunicación. Desde esta concepción se entiende el discurso como práctica social:

Hablar de discurso es, ante todo, hablar de una práctica social, de una forma de acción entre las personas que se articula a partir del uso lingüístico contextualizado, ya sea oral o escrito. El discurso es parte de la vida social y a la vez un instrumento que crea la vida social. Desde el punto de vista discursivo, hablar o escribir no es otra cosa que construir piezas textualesorientadas a unos fines y que se dan en interdependencia con el contexto (lingüístico, local, cognitivo y sociocultural) (Calsamiglia y Tusón, 2002: 15).

Esta concepción nos remite a abordar la importancia del discurso en la sociedad contemporánea. Santander (2011: 208) lo explica a partir de cuatro instancias:

-La importancia analítica de los discursos, (interés de las Ciencias Sociales por el estudio de los discursos debido a la valoración del lenguaje y la concepción teórico-metodológica que han adquirido los estudios del discurso)

-La doble hermenéutica expresada en la relevancia que ha adquirido la noción de discurso y en la toma de conciencia que se ha adquirido ante el hecho de que la mayoría de los investigadores se enfrentan a signos de diversa naturaleza que requieren ser leídos para su correcta interpretación. "(...) Los investigadores producen textos acerca de esos textos en una suerte de doble hermenéutica".

-La opacidad de los signos. Al respecto Santander plantea: "Ya no basta explicar la comunicación humana como un proceso de codificación y decodificación pues ésta tiene un componente fuertemente inferencial, lo que significa que a menudo importa más la inferencia que los signos provocan que el significado literal de ellos, o sea, las palabras significan mucho más de lo que dicen".

- El discurso como dimensión crucial en el establecimiento de los vínculos y de las relaciones sociales y políticas (luchas a favor del reconocimiento de las diferencias, problemática cultural e identitaria). 
Según Ibáñez,

la dicotomía mente/mundo es reemplazada por la dualidad discurso/mundo. (...) Lo social como objeto de observación no puede ser separado ontológicamente de los discursos que en la sociedad circulan. Estos discursos, además y a diferencia de las ideas, son observables y (...) constituyen una base empírica más certera que la introspección racional (2003).

Todo lo anterior permite afirmar que el conocimiento del mundo no radica en las ideas, sino en los enunciados que circulan. Como vemos, este paradigma le reconoce al lenguaje una función no sólo referencial (informativa) y epistémica (interpretativa), sino también realizativa (creativa), o, generativa (Echevarría, 2003).

En este sentido es importante lo siguiente:

Los discursos llevan consigo contenidos, significados de orden ideológico, cultural y modos de posicionarse y actuar en el mundo. Tienen su origen en las prácticas institucionales y en las relaciones de poder, por lo que se convierten en una de las formas más usuales de producción, circulación, recepción y en esencia de reproducción de los sistemas de creencias de los grupos sociales que son transmitidos en el contexto de las prácticas semiótico-discursivas de las que estos forman parte (Pérez Terán, 2008: 97).

A partir de lo anterior es posible comprender que las prácticas comunicativas de los escenarios contemporáneos se viabilizan en torno a la concepción de que "toda práctica o praxis social es una práctica semiótico- discursiva y está determinada por una lucha de clases, en un momento histórico específico (...). En una práctica social, los discursos se refieren a lo que puede ser dicho y pensado, pero también a quién puede hablar, cuando y con qué autoridad en el marco de dicha práctica" (Pérez Terán, 2008: 96-97).

\section{Las prácticas discursivas}

Los discursos como prácticas discursivas, son acontecimientos discursivos que tienen un espesor y una importancia tan grande como la de los acontecimientos no discursivos (Haidar, 1998: 134), puesto que su ámbito no se restringe únicamente al espacio de lo dicho, sino que también propone, reconfigura y establece pautas de comportamiento. 
Partiendo de lo anterior, entendemos la categoría de prácticas discursivas para hacer referencia a toda aquella que se sostiene en el uso contextualizado del discurso. Al asumir esta concepción, se trabaja con una categoría general que engloba las distintas modalidades de estudio del discurso, dígase lo escrito, lo oral, lo visual y lo audiovisual. La práctica discursiva implica la construcción de la identidad y su subjetividad, en la que el discurso en sí mismo permite entender a los actores sociales como construcciones (Labarta, 2010: 206).

Las prácticas discursivas presentan las siguientes características, siguiendo a Haidar (2000: 57):

1. Están antes, durante o después de cualquier práctica socio-cultural-histórica;

2. Producen, reproducen y transforman la vida social en todas sus dimensiones;

3. Tienen una función performativa, es decir, pueden producir diferentes tipos de prácticas socio-culturales;

4. Son en sí mismas prácticas socio-culturales;

5. Producen y reproducen, de diversas maneras, las distintas materialidades que las construyen;

6. Son importantes para la producción y reproducción de la hegemonía y el poder, por ello sirven para accionar los mecanismos de persuasión;

7. En ellas también pueden originarse procesos de resistencia y de lucha contra la dominación y la explotación.

\section{Implicaciones epistemológicas, teóricas y metodológicas de considerar la categoría prácticas discursivas en la investigación en comunicación social}

Como se ha visto, "abordar los discursos como prácticas discursivas implica un giro analítico significativo que nos ubica directamente en la dimensión pragmática" (Haidar, 2000: 57). Teniendo en cuenta, entonces, la relevancia social que adquiere el ejercicio profesional de la comunicación cuyos predios se sitúan en el espacio de lo público, en el establecimiento de diálogo, en una gestión eficaz de los procesos de comunicación en las organizaciones, tanto en la comunicación interna como externa; en la creación de consenso, en la contribución a la pervivencia de ideologías, culturas, formas simbólicas; así como al combate contra las formas hegemónicas de producción comunicativa, el discurso precisa entonces no solo de su capacidad enunciativa, sino también de la capacidad de hacer. 
En el ámbito de estudio de la comunicación las prácticas discursivas se concretan en la realización de discursos para el desarrollo, discursos políticos, discursos históricos, discursos de género, discursos académicos, científicos, discursos medioambientales, discursos institucionales, publicitarios, discursos mediáticos, discursos sindicales entre otros que circulan en la sociedad, en los que los actores de la comunicación representan posturas ideológicas, políticas, sociales, en general. Dada la naturaleza de estas prácticas discursivas, resultan relevantes para el campo de la comunicación en dos sentidos: 1. En tanto expresión concreta del proceso de la comunicación, y por ende, en el ejercicio más importante del profesional de la comunicación y 2. Como fuente de información en torno a la realidad social en que se encuentra inmerso este profesional, donde quizá se ubique el problema a solucionar o la propuesta a realizar.

Desde este punto de vista, la investigación de las prácticas discursivas ha sido vasta, a partir del acercamiento de múltiples disciplinas, dígase el análisis literario, análisis semiótico, etnometodología, lingüística del texto, análisis del discurso, entre otros. La tradición de estudios relacionada con el análisis de los discursos, privilegia un enfoque centrado en el desmontaje de las estrategias y estructuras de reproducción ideológicos en los discursos de los medios de comunicación, con estrecho vínculo con las condicionantes socioculturales e institucionales que la transversalizan en un nivel macro; con énfasis en la concepción de discurso como suma de texto más contexto.

La concepción de prácticas discursivas contiene en sí misma una referencia a uno de los componentes que forman parte de todo proceso comunicativo y al que aludíamos al inicio: los actores de la comunicación, tanto en su condición de emisor como de receptor. De manera que el análisis de las prácticas discursivas debería contemplar el estudio de las siguientes dimensiones en su conjunto: actores (emisor/receptor), texto/discurso y contexto.

De esta idea general se deriva la siguiente aproximación a las implicaciones epistemológicas, teóricas y metodológicas que deberían ser contempladas en la investigación de las prácticas discursivas en el campo de la comunicación social.

Desde el punto de vista epistemológico habría que considerar: una concepción de la investigación en la que se articulen, desde la planeación misma, el abordaje de la práctica discursiva en todas las dimensiones del ciclo comunicativo; es decir que contemple las condiciones de la emisión o producción, con énfasis en los actores; la expresión (discurso) y los factores relacionados con la recepción, en tanto los actores que toman parte de esta actividad constituyen entes de relevancia para comprender el sentido último apropiado. 
Contemplar las condiciones de producción, circulación y recepción de los discursos sería estratégico.

Desde la visión teórica habría que tomar en cuenta: la diversificación de las perspectivas teóricas con las que se observa el objeto, teniendo en cuenta la interdisciplinariedad, inherente a este tipo de investigación y al objeto de la comunicación en general. En este sentido habría que tomar en cuenta los aportes de la Psicología, el Interaccionismo simbólico, la Sociología de los emisores, la Pragmática, los estudios de la propaganda, la publicidad, la economía política de la comunicación, los estudios de recepción, entre otros.

Desde la concepción metodológica habría que considerar que: el empleo de herramientas de análisis que superen el límite del análisis discursivo o textual y tome en cuenta otras herramientas como: grupos de discusión, las técnicas proyectivas, entrevistas, encuestas, entre otras, que permitirían estudiar los actores de la comunicación.

\section{Conclusiones}

La investigación del discurso desde la concepción de prácticas discursivas en el ámbito de la Comunicación Social ensancha desde el punto de vista epistemológico, teórico y metodológico, el abordaje de esta temática; fundamentalmente en los escenarios de la comunicación actual en los que las nuevas tecnologías aportan un componente importante a tener en cuenta, toda vez que los sujetos o actores adquieren un papel activo en los procesos discursivos y sociales. La complejidad de estos procesos propicia que los enfoques de la investigación en comunicación social deban repensarse a la luz de las coyunturas sociales y de lo que demandan los escenarios sociales.

\section{Notas}

(1) Dentro de la producción científica deben considerarse aquellos trabajos investigativos en proceso o el resultado de procesos de culminación de estudios o de superación postgraduada. Específicamente, entendemos como producción científica la literatura gris que es el "conjunto de documentos de tirada limitada y circulación restringida que no pueden obtenerse por canales habituales de venta" (Piedra, 2005: 30). Consideramos dentro de este grupo a los Trabajos de Diploma, Tesis de Maestría y Doctorados.

(2) La Facultad de Comunicación de la Universidad de La Habana cuenta con tres carreras de pregrado: Ciencias de la Información, Periodismo y Comunicación Social y en el postgrado con los siguientes programas: Maestría y doctorado en Ciencias de la Información y Ciencias de la Comunicación. 


\section{Bibliografía}

Alonso, M. y Saladrigas, H. (2000). Para investigar en Comunicación Social. Guía didáctica. Cuba: Editorial Pablo de la Torriente Brau.

Calsamiglia, H. y Tusón, A. (2002). Las cosas del decir. Manual de análisis del discurso. España: Ariel.

Candela, A. (1999). Prácticas discursivas en el aula y calidad educativa. Revista Mexicana de Investigación Educativa, 4(8), 273-298. Recuperado de http://www.redalyc.org/pdf/140/14000804.pdf.

Dijk van, T. A. (1992). La ciencia del texto. Barcelona: Paidós.

Echevarría, R. (2003). Ontología del lenguaje. Santiago: J. C. Editor.

Galindo, J. (2008). Comunicación, ciencia e historia. Fuentes científicas históricas hacia una Comunicología posible. Madrid: McGraw-Hill/Interamericana de España.

Haidar, J. (1998). Análisis del discurso. En Galindo, J. Técnicas de investigación en sociedad, cultura y comunicación (pp. 117-164). Mexico: Addison Wesley Longman.

Haidar, J. (2000). El poder y la magia de la palabra: El campo de análisis del discurso. En La producción textual del discurso científico. México: Universidad Autónoma Metropolitana.

Ibañez, T. (2003). El giro linguístico. En Análisis del discurso. Manual para las Ciencias sociales (pp. 21-42). Barcelona: UOC.

Karam, T. (2005). Introducción al Estudio del discurso y Análisis del discurso. Global Media Journal, 2(3). Recuperado de www.redalyc.or

Labarta, M. (2010). Análisis crítico de la prácticas discursivas en torno al plan de reforma de la Muralla Ärabe en el Centro histórico de Valencia. Representación de los actores implicados. Arxius de Ciencias Sociales, 23, 215-212. Recuperado de http://lamuralladelcarmen.blogspot.com/p/analisis-critico-de-las-practicas.html

Lugones, M. (2016). La investigación en Comunicación Social en Cuba: un articulación desde los presupuestos institucionales, cognoscitivos y culturales. (Tesis inédita de doctorado). Departamento de Comunicación Social. Facultad de Comunicación. Universidad de La Habana.

Martín-Serrano, M. (1982). Propuesta de un modelo dialéctico para el estudio de los sistemas de comunicación. En Saladrigas, H. (Comp.) (2006). Introducción a la teoría e investigación en Comunicación (pp. 99-113). La Habana: Editorial Ciencias Médicas. 
Pérez Terán, J. (2008). Las prácticas discursivas institucionalizadas. Lingua Americana, XII(22), $95-110$

http://www.produccioncientifica.luz.edu.ve/index.php/lingua/article/download/17206/171 79

Pérez, M. (diciembre de 2008). Discusiones teóricas y metodologicas sobre el estudio del discurso desde el campo de la comunicación. Comunicación y sociedad, 10, (pp.225247).

Recuperado

Http://www.scielo.org.mx/scielo.php?script=sci_arttext\&pid=S0188-

$52 X 2008000200009 \&$ Ing $=$ es\&nrm=iso>

Piedra, Y. (2009). La producción científica sobre comunicación: análisis desde una perspectiva de dominio en la Web de la ciencia. Periodo 2000-2007. (Tesis inédita de maestría en Ciencias de la Información). Facultad de Comunicación, Universidad de La Habana.

Saladrigas Medina, H. (2005). Coordenadas cubanas para un fenómeno complejo: Fundamentos para un enfoque teórico-metodológico de la investigación de la Comunicación Organizacional. (Tesis inedita de Doctorado). Facultad de Comunicación, Universidad de La Habana.

Santander, P. (2011). ¿Por qué y cómo hacer análisis del discurso? Cinta Moebio, 41, 207-224. doi:www.moebio.uchile.cl/41/santander.html

Vázquez, Y. y Nápoles L. (2015). La investigación en comunicación social en la Facultad de Comunicación de la Universidad de La Habana, vista desde su vínculo con el análisis del discurso. VIII Encuentro Internacional de Investigadores y Estudiosos de la Información y la Comunicación, ICOM 2015, Cuba.

Vázquez, Y. (2015). Acercamiento a la producción científica centrada en el análisis del discurso, durante el período 2005- 2014, en la Facultad de Comunicación de la Universidad de La Habana. (Tesis inédita de Maestría). Facultad de Comunicación, Universidad de la Habana. 